\title{
Reabsorción Condilar como Complicación Postoperatoria en Pacientes Sometidos a Cirugía Ortognática. Revisión Narrativa
}

\author{
Condylar Resorption as a Postoperative Complication in Patients \\ Undergoing Orthognathic Surgery. Narrative Review
}

\author{
Javier Zerené Reyes'; María José Zilleruelo Pozo'; Miguel Vergara Iturriaga'; \\ Alfredo Noguera Pantoja² \& Pedro Solé Ventura ${ }^{2}$
}

\begin{abstract}
ZERENÉ, R. J.; ZILLERUELO, P. M. J.; VERGARA, I. M.; NOGUERA, P. A. \& SOLÉ, V. P. Reabsorción condilar como complicación postoperatoria en pacientes sometidos a cirugía ortognática. Revisión narrativa. Int. J. Odontostomat., 13(3):251257, 2019.

RESUMEN: La reabsorción condilar como complicación postoperatoria en cirugía ortognática es una causa frecuente de recidiva de anomalías dentomaxilares, existiendo diversos factores que se relacionan con su aparición. El objetivo de este estudio fue describir mediante una revisión narrativa la reabsorción condilar como complicación postoperatoria en cirugía ortognática. Se realizó una búsqueda electrónica de la literatura en las bases de datos electrónicas PubMed, EBSCO, TripDatabase y Epistemonikos sin límite de años, en idioma inglés y español, incluyendo revisiones sistemáticas, ensayos clínicos y estudios observacionales. Se excluyeron reportes de casos, estudios en animales y aquellos que no relacionaran la complicación con cirugía ortognática. Se evaluaron los estudios según grado de recomendación y calidad de reporte. Veintiún artículos fueron seleccionados según los criterios de selección establecidos en esta revisión. La literatura reportada sugiere que la reabsorción condilar es una patología de frecuencia relativa en pacientes postoperados de cirugía ortognática (1,4-32 \% de los casos) y que está asociada a factores de riesgo preoperatorios tales como género, edad, tipo de anomalía dentomaxilar y técnica quirúrgica utilizada. La reabsorción condilar es una complicación postoperatoria a cirugía ortognática que debemos considerar en la planificación del tratamiento e identificar pacientes con factores de riesgo. Luego de la intervención quirúrgica es de vital importancia realizar un seguimiento estricto a este tipo de pacientes e identificar de forma temprana cambios clínicos y radiográficos. Finalmente, es importante seguir investigando sobre esta materia para establecer criterios de prevención y diagnóstico, con mayor claridad.
\end{abstract}

PALABRAS CLAVE: cirugía ortognática, cóndilo mandibular, articulación temporomandibular.

\section{INTRODUCCIÓN}

La cirugía ortognática corresponde a un procedimiento quirúrgico, cuyo objetivo principal es devolver o mejorar la función del sistema estomatognático, incluyendo función masticatoria, fonación, función respiratoria e incluso como tratamiento de apnea obstructiva del sueño (Fish et al., 1993; Te Veldhuis et al., 2017). A pesar de que la cirugía ortognática hoy se considera un procedimiento exitoso y de bajo riesgo quirúrgico, la literatura plantea que cerca del $40 \%$ de los pacientes que se someten a cirugía ortognática presentan complicaciones ya sea pre, intra o postoperatorias. Dentro de las complicaciones postoperatorias asociadas a la articulación témporomandibular, encontramos la reabsorción condilar (Te Veldhuis et al.).

La reabsorción condilar post-quirúrgica corresponde a una alteración progresiva de forma y volumen en los cóndilos mandibulares secundario a una osteotomía sagital de rama mandibular bilateral (OSRMB), cirugía bimaxilar u osteotomía maxilar tipo Le Fort I (Hoppenreijs et al., 1998), lo que lleva a desarrollar características clínicas tales como disminución de la altura facial posterior, retrusión mandibular

\footnotetext{
${ }^{1}$ Cirujano Dentista, Universidad de los Andes, Santiago, Chile.

${ }^{2}$ Cirujano Dentista, Cirujano Maxilofacial, Universidad de los Andes, Santiago, Chile.
} 
ZERENÉ, R. J.; ZILLERUELO, P. M. J.; VERGARA, I. M.; NOGUERA, P. A. \& SOLÉ, V. P. Reabsorción condilar como complicación postoperatoria en pacientes sometidos a cirugía ortognática. Revisión narrativa. Int. J. Odontostomat., 13(3):251-257, 2019.

progresiva y mordida abierta anterior. Esta alteración postoperatoria debe ser diferenciada de reabsorciones condilares asociadas a factores locales como trauma, tumores o malformaciones, o de causas sistémicas ya sea, inflamatorias o infecciosas (Alexiou et al., 2009).

El objetivo de esta revisión es describir la reabsorción condilar como complicación postoperatoria en pacientes sometidos a cirugía ortognática, cuáles son sus métodos diagnósticos y los factores de riesgo que predisponen a la aparición de esta condición.

\section{MATERIAL Y MÉTODO}

Se realizó una búsqueda electrónica de la literatura en las siguientes bases de datos: Pubmed, EBSCO, Trip Database y Epistemonikos. Se utilizaron las siguientes palabras claves: MeSH "orthognathic surgery", "mandibular advancement", "bimaxillary surgery", "bilateral sagittal split osteotomy", "bimaxillary osteotomy", "vertical ramus osteotomy", "le fort I", "condylar resorption", "idiopathic condylar resorption", "progressive condylar resorption", "condylar atrophy", "condylolysis", combinados con los operadores booleanos AND y OR. Los criterios de inclusión fueron artículos en idioma inglés o español, sin límite de años, disponibles en texto completo, incluyendo revisiones sistemáticas, ensayos clínicos y estudios observacionales. Se consideraron como criterios de exclusión artículos basados en reportes de casos, estudios en animales y estudios que no relacionaran la reabsorción condilar con cirugía ortognática. Adicionalmente, se llevó a cabo una búsqueda retrógrada de artículos en las referencias de la literatura seleccionada. Para determinar el nivel de evidencia y el grado de recomendación se utilizaron las pautas de Oxford Centre for Evidence-Based Medicine.

\section{RESULTADOS Y DISCUSIÓN}

Se obtuvieron 129 artículos luego de realizar la búsqueda electrónica, sin aplicar filtros en ninguna base de datos. De ellos 33 artículos se encontraban repetidos. Tras aplicar criterios de inclusión y exclusión y realizar la búsqueda retrógrada, los artículos seleccionados fueron 21 (Fig. 1).

En la selección de pacientes para los estudios observacionales revisados no existen grandes diferen- cias en cómo los autores determinan los criterios de inclusión, excepto en los estudios de Nogami et al. (2016) y Gomes et al. (2017) que aclaran que los pacientes incluidos eran pacientes de sexo femenino que presentaban osteoartritis previa. En relación a los criterios de exclusión, los estudios presentan en general los mismos parámetros, excluyendo pacientes que presentaran síndromes de las bases craneales (fisuras labio palatinas, por ejemplo), enfermedades sistémicas de carácter degenerativo o intervenciones previas en la articulación temporomandibular (Kerstens et al., 1990; Merkx \& Van Damme, 1994; de Mol van Otterloo et al., 1993; Bouwman et al., 1994, 1997; De Clercq et al., 1994; Scheerlinck et al., 1994; Cutbirth et al., 1998; Hoppenreijs et al., 1998; Hwang et al., 2000a,b, 2004; Wolford et al., 2002, 2003; Borstlap et al., 2004; Wohlwender et al., 2011; Kobayashi et al., 2012; Xi et al., 2015; Nogami et al.; Gomes et al.).

Al considerar todos los artículos incluidos se observó una prevalencia de esta complicación entre un $1,4 \%$ a un $32 \%$ (Wolford et al., 2002; Nogami et al.), lo que arroja como promedio un 10,6 \% (Merkx \& Van Damme; Kerstens et al.; de Mol van Otterloo et al.; Bouwman et al., 1994, 1997; De Clercq et al.; Scheerlinck et al.; Cutbirth et al.; Hoppenreijs et al., 1998; Hwang et al. 2000a,b, 2004; Wolford et al., 2002, 2003; Borstlap et al.; Wohlwender et al.; Kobayashi et al.; Xi et al.; Nogami et al.; Gomes et al.). Se analizaron los estudios seleccionados (Tabla I) con el objetivo de describir los factores de riesgo post-quirúrgicos para presentar esta complicación, analizando las características con que se presenta y su frecuencia en la literatura. La reabsorción condilar posterior a una cirugía ortognática es una complicación postoperatoria que se debe tener en cuenta. Hay diversos factores de riesgo descritos que se asocian a su aparición y/o progresión. Entre ellos se encuentran:

\section{Factores de riesgo clínico - demográficos}

Sexo: todos los estudios observacionales analizados que expresan el sexo en cual ocurre la reabsorción condilar presentan una mayor prevalencia en mujeres versus hombres en una relación de 15,5:1 (Merkx \& Van Damme; De Clercq et al.; Scheerlinck et al.; Bouwman et al., 1997; Cutbirth et al.; Hoppenreijs et al., 1998, 1999; Hwang et al., 2000a,b 2004; Borstlap et al.; Kobayashi et al.; Nogami et al.; Gomes et al.). Dos estudios especifican que las diferencias fueron estadísticamente significativas (Hoppenreijs et al., 1998; Borstlap et al.). Se cree que estaría más asociada a pacientes de género femenino debido a la 


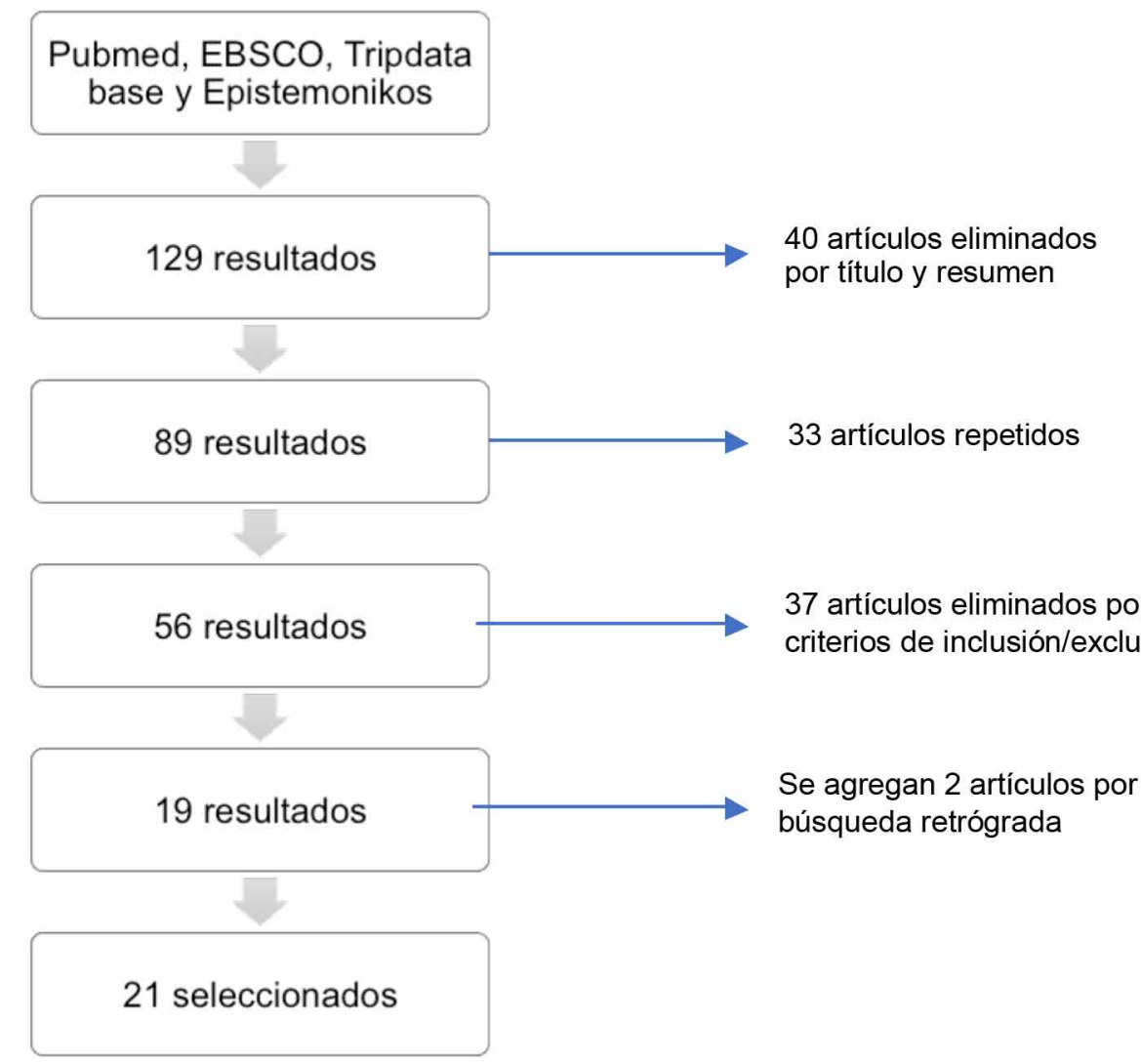

Fig. 1. Diagrama de flujo para la selección de artículos incluidos en la revisión.

Tabla I. Estudios incluidos en la revisión.

\begin{tabular}{|c|c|c|c|c|}
\hline Autor & Año & Diseño Del Estudio & $\begin{array}{l}\text { Nivel de } \\
\text { Evidencia }\end{array}$ & $\begin{array}{c}\text { Grado de } \\
\text { Recomendación }\end{array}$ \\
\hline Kertens et al. & 1990 & Observacional retrospectivo. & 4 & $\mathrm{C}$ \\
\hline De mol van otterloo et al. & 1993 & Observacional retrospectivo. & 4 & C \\
\hline Merkx et al. & 1994 & Observacional retrospectivo. & 4 & $\mathrm{C}$ \\
\hline Bouwman et al. & 1994 & Observacional retrospectivo. & 4 & C \\
\hline Scheerlinck et al. & 1994 & Observacional prospectivo & 4 & C \\
\hline De clercq et al. & 1994 & Observacional retrospectivo. & 4 & $\mathrm{C}$ \\
\hline Bouwman et al. & 1997 & Casos y controles. & $3 b$ & B \\
\hline Hoppenreijs et al. & 1998 & Observacional retrospectivo. & 4 & $\mathrm{C}$ \\
\hline Cutbirth et al. & 1998 & Observacional retrospectivo. & 4 & $\mathrm{C}$ \\
\hline Hoppenreijs et al. & 1999 & Observacional retrospectivo. & 4 & $\mathrm{C}$ \\
\hline Hwang et al. & 2000 & Observacional retrospectivo. & 4 & $\mathrm{C}$ \\
\hline Hwang et al. & 2000 & Casos y controles. & $3 b$ & $\mathrm{~B}$ \\
\hline Wolford et al. & 2002 & Observacional retrospectivo. & 4 & C \\
\hline Wolford et al. & 2003 & Observacional retrospectivo. & 4 & C \\
\hline Hwang et al. & 2004 & Casos y controles. & $3 b$ & B \\
\hline Borstlap et al. & 2004 & Observacional prospectivo. & 4 & C \\
\hline Wohlwender et al. & 2011 & Observacional retrospectivo. & 4 & C \\
\hline Kobayas hi et al. & 2012 & Observacional retrospectivo. & 4 & C \\
\hline Xi et al. & 2015 & Observacional prospectivo. & 4 & C \\
\hline Nogami et al. & 2016 & Casos y controles. & $3 b$ & B \\
\hline Gomes et al. & 2017 & Observacional retrospectivo. & 4 & $\mathrm{C}$ \\
\hline
\end{tabular}


ZERENÉ, R. J.; ZILLERUELO, P. M. J.; VERGARA, I. M.; NOGUERA, P. A. \& SOLÉ, V. P. Reabsorción condilar como complicación postoperatoria en pacientes sometidos a cirugía ortognática. Revisión narrativa. Int. J. Odontostomat., 13(3):251-257, 2019.

modulación de hormonas como el estrógeno y la prolactina, así como en el metabolismo del fibrocartílago y diferencias en el aporte sanguíneo que contribuirían a un mayor riesgo de reabsorción (Hoppenreijs et al., 1998; Borstlap et al.).

Edad: el promedio de edad de los pacientes que presentaron reabsorción condilar fue de 22,6 años (14 a 39 años), siendo la década de los veinte años la más predominante en la distribución (Merkx \& Van Damme; de Mol van Otterloo et al.; Scheerlinck et al.; Bouwman et al., 1997; Hoppenreijs et al., 1999; Hwang et al., 2000a,b, 2004; Kobayashi et al.; Nogami et al.). Diez estudios no especificaban la edad en la que fueron intervenidos los pacientes que presentaron reabsorción condilar (Kerstens et al.; Bouwman et al., 1994; De Clercq et al.; Hoppenreijs et al., 1998; Wolford et al., 2002, 2003; Borstlap et al.; Wohlwender et al.; Xi et al.; Gomes et al.).

Anomalía dentomaxilar: dentro de las anomalías que presentaban los pacientes intervenidos, se observó en un $38 \%$ de los casos hipoplasia mandibular (Bouwman et al., 1994; Scheerlinck et al.; Hoppenreijs et al., 1998; Hwang et al., 2000a,b, 2004; Kobayashi et al.; Xi et al.); retrognasia mandibular en el $28,5 \%$ de los casos (Kerstens et al.; de Mol van Otterloo et al.; De Clercq et al.; Bouwman et al., 1997; Nogami et al.; Gomes et al.); anomalías clase II esqueletal en el estudio de Merkx \& Van Damme y clase II dentaria sin especificar alteraciones en bases craneales (Wolford et al., 2002). Dos estudios incluyeron pacientes con laterognasia (Wohlwender et al.; Merkx \& Van Damme). En cuanto a si estas anomalías se asocian a un mayor riesgo de reabsorción condilar postoperatorio, Bouwman et al. (1994) y Hwang et al. (2004) establecen de forma estadísticamente significativa a la hipoplasia mandibular como factor predisponente para el desarrollo de reabsorción condilar postoperatoria. Sólo Scheerlinck et al. aclaran que la anomalía dentomaxilar no tiene significancia estadística como para relacionarla con la condición adquirida.

Ángulo del plano mandibular: se define como el ángulo formado por el plano mandibular y la línea silla - nasion (S-N) en el estudio cefalométrico (Xi et al.). Esta característica varía según la población en la que son estudiados los pacientes y todos los estudios aclaran que las mediciones fueron realizadas según la población general a la que pertenecían los pacientes (Kerstens et al.; de Mol van Otterloo et al.; Bouwman et al., 1994, 1997; De Clercq et al.; Scheerlinck et al.; Cutbirth et al.; Hoppenreijs et al.,
1998, 1999; Hwang et al., 2000a,b, 2004; Borstlap et al.; Xi et al.; Nogami et al.; Gomes et al.). Catorce artículos de los revisados, correspondiente al 63,6 $\%$, especifican que los pacientes tenían amplio ángulo del plano mandibular. El número varía según los estudios, pero en general se considera un plano mandibular alto entre $30-50^{\circ}$. Sólo seis artículos consideran esta característica como estadísticamente significativa para desarrollar reabsorción condilar (Bouwman et al., 1994; Gomes et al.; Hwang et al., 2004; Xi et al.; Nogami et al.). Según esto, la retrognasia mandibular, hipoplasia mandibular y alto ángulo del plano mandibular son anomalías dentomaxilares susceptibles al desarrollo de esta complicación post-cirugía ortognática.

Inclinación del cuello condilar: Hwang et al. (2000a,b) evaluó a 240 pacientes clase II sometidos a cirugía ortognática, donde once de ellos presentaron reabsorción condilar postoperatoria y todos tenían inclinación posterior del cóndilo pre-operatoria. Se propone que, al existir una inclinación posterior del cuello condilar, la superficie anterosuperior queda desprovista de carga y por lo tanto, es menos densa. Esto genera una mayor susceptibilidad a generar reabsorción condilar, cuando se genera rotación del cóndilo o movimientos en sentido cefálico en el procedimiento quirúrgico.

Trastornos temporomandibulares (TTM): la presencia de síntomas preoperatorios de trastornos temporomandibulares se ha considerado a lo largo de los años como un factor predisponente para desarrollar reabsorción condilar (Cutbirth et al.; Wolford et al., 2003; Borstlap et al.). Sin embargo, no existe un consenso sobre esta aseveración. Sólo once artículos se refieren a las condiciones articulares que presentaban los pacientes inicialmente (Merkx \& Van Damme; Kerstens et al.; de Mol van Otterloo et al.; De Clercq et al.; Scheerlinck et al.; Cutbirth et al.; Hoppenreijs et al.,1998, 1999; Hwang et al., 2004; Kobayashi et al.; Nogami et al.). Dos autores no encontraron influencia estadísticamente significativa para el desarrollo de reabsorción condilar (De Clercq et al.; Hwang et al., 2004) y sólo Cutbirth et al. plantearon diferencias estadísticamente significativas con la influencia de TTM previos para el desarrollo de reabsorción condilar. Wolford et al. describen que en caso de realizar una cirugía de reposicionamiento discal de ATM, ésta debe ser en conjunto o antes que se realice cirugía ortognática, ya que así se pueden predecir mejor los resultados de estabilidad de la ATM (Wolford et al., 2002). 
ZERENÉ, R. J.; ZILLERUELO, P. M. J.; VERGARA, I. M.; NOGUERA, P. A. \& SOLÉ, V. P. Reabsorción condilar como complicación postoperatoria en pacientes sometidos a cirugía ortognática. Revisión narrativa. Int. J. Odontostomat., 13(3):251-257, 2019.

\section{Factores de riesgo intraoperatorios}

Tipo de osteotomía realizada: los reportes encontrados en la literatura demuestran que existe un riesgo de que se produzca reabsorción condilar al realizar osteotomías tanto en maxilar como mandíbula o la combinación de ambas. Sin embargo, en la literatura estudiada la mayor prevalencia se asocia a cirugías bimaxilares $(64,1 \%)$, luego asociada a osteotomía sagital de rama mandibular bilateral (OSRMB) en el 28,2 $\%$ de los casos, en osteotomías maxilares tipo Le Fort I en el $6,4 \%$ y asociada a una osteotomía sagital de rama mandibular unilateral (OSRMU) en el 1,2 \% de los pacientes intervenidos. En el caso de Wohlwender et al., no se observaron diferencias estadísticamente significativas entre OSRMB y OSRMU. Tampoco se observó diferencia en la predisposición por el lado intervenido y la incidencia de reabsorción condilar (Merkx \& Van Damme; Kerstens et al.; de Mol van Otterloo et al.; Bouwman et al., 1994, 1997; De Clercq et al.; Scheerlinck et al.; Cutbirth et al.; Hoppenreijs et al., 1998, 1999; Hwang et al., 2000a,b, 2004; Wolford et al. 2002, 2003; Borstlap et al.; Wohlwender et al.; Kobayashi et al.; $\mathrm{Xi}$ et al.; Gomes et al.). Cinco estudios presentaron resultados estadísticamente significativos entre el grado de avance mandibular y la incidencia de reabsorción condilar (Scheerlinck et al.; Cutbirth et al.; Borstlap et al.; Nogami et al.; Gomes et al.). En el caso de una OSRM, se producen cargas mecánicas durante y después de la intervención, que generan el impedimento de flujo nutricio al cóndilo y cápsula articular. El incremento en la carga articular es mayor en casos de rotaciones anti horarias o avances mandibulares de gran magnitud, por lo que debemos considerar que estos pacientes podrían tener una mayor predisposición a presentar reabsorciones como complicación de la cirugía. Al utilizar una osteotomía tipo Le Fort I como técnica única se observan menos casos de reabsorción condilar postoperatoria (Hoppenreijs et al., 1998; Hwang et al., 2000a,b). No obstante, la heterogeneidad de los estudios en cuanto a comparar todas las técnicas entre sí sin considerar la frecuencia con la que se desarrolla cada una, hace que no podamos llegar a una conclusión definitiva.

Osteosíntesis y fijación intermaxilar (FIM): en los estudios más antiguos de esta revisión se menciona el uso de fijación o bloqueo intermaxilar alámbrico postoperatorio, aparatos de ortodoncia y arcos de barra (Merkx \& Van Damme Kerstens et al.; de Mol van Otterloo et al.; Bouwman et al., 1994, 1997; Scheerlinck et al.; Hoppenreijs et al., 1998, 1999; Hwang et al., 2000a,b, 2004; Kobayashi et al.; Xi et al.). Sin embargo, actualmente la fijación intermaxilar queda relegada a ciertos procedimientos quirúrgicos, como la osteotomía vertical de rama mandibular (OVRM) (Jung et al., 2012). De hecho, Bouwman et al. (1994), menciona cómo influye la fijación intermaxilar en el desarrollo de la complicación al afectar de forma negativa la función de la ATM, por restricción en la función normal y probable disminución en el aporte nutricio que generan los cambios en la relación cóndilo - fosa. En los resultados del estudio de Hoppenreijs et al. se asocia de forma estadísticamente significativa la osteotomía tipo Le Fort I con la fijación intermaxilar alámbrica y mayor incidencia de reabsorción condilar (Hoppenreijs et al., 1998). En dos estudios se realizó osteosíntesis rígida con placas de titanio o tornillos bicorticales (Cutbirth et al.; Wohlwender et al.) y en 6 estudios no se especifica si se realizó fijación intermaxilar en los pacientes intervenidos (De Clercq et al.; Hoppenreijs et al., 1999; Wolford et al., 2002, 2003; Borstlap et al.; Gomes et al.). En los casos de fijación interna rígida, la circulación del fluido sinovial y de los aportes nutricios se ve menos comprometida y las capacidades de adaptación serían mejores que en los grupos que se utiliza fijación intermaxilar (Hoppenreijs et al., 1998; Hwang et al., 2004). Scheerlinck et al. plantean mejores resultados en pacientes donde se realiza fijación interna rígida (Scheerlinck et al.; Hoppenreijs et al., 1998) y Hwang et al. $(2000 a, b)$ no encontró diferencias estadísticamente significativas en los tipos de fijación de osteosíntesis o la aplicación de fijación intermaxilar y la presencia de reabsorción condilar.

Características de la reabsorción condilar post quirúrgica. En los estudios analizados se menciona que los rangos de pérdida de tejido condilar fueron entre -2 a - $8 \mathrm{~mm}$. Scheerlinck et al. indican que 4 de los 8 pacientes generaron una reabsorción completa de los cóndilos mandibulares y 10 estudios observacionales no especifican las superficies afectadas por la reabsorción condilar. En relación a la ubicación anatómica de esta complicación, a pesar de realizar la misma técnica quirúrgica de manera bilateral, la reabsorción condilar tiende a ser unilateral con mayor frecuencia. De hecho, en el $56,9 \%$ de los casos en los que se presentó reabsorción condilar posterior a osteotomía sagital de rama bilateral, ésta fue unilateral.

Los autores plantean que los primeros signos y síntomas se presentan dentro de los primeros 6 meses postoperatorios, siendo fundamental el seguimiento en el diagnóstico de esta condición. Hoppenreijs et al. muestra que al año postoperatorio se encontraron 18 casos de reabsorción condilar y en el seguimiento a largo plazo, que fue en promedio de 5,9 años, el núme- 
ZERENÉ, R. J.; ZILLERUELO, P. M. J.; VERGARA, I. M.; NOGUERA, P. A. \& SOLÉ, V. P. Reabsorción condilar como complicación postoperatoria en pacientes sometidos a cirugía ortognática. Revisión narrativa. Int. J. Odontostomat., 13(3):251-257, 2019.

ro de pacientes que presentaron esta condición aumentaron a 40 de un total de 259 (Hoppenreijs et al., 1998). El seguimiento más alto registrado en los estudios fue de diez años, en el de Hoppenreijs et al. (1999). Se evidenció que hubo un rango de $25-150 \%$ de recidiva de las condiciones logradas por la cirugía. Particularmente, el estudio de Hoppereijs, et al., muestra las tasas de recidiva más altas, pero sólo los relaciona con el tipo de cirugía y no menciona factores de riesgo preoperatorios (Hoppenreijs et al., 1999).

\section{Métodos diagnósticos}

En cuanto a los métodos diagnósticos para identificar esta complicación encontramos parámetros clínicos e imagenológicos. En cuanto a los criterios clínicos debemos evaluar cambios a nivel facial y oclusal (Tabla II). Dependiendo de la ubicación en la que se presente la reabsorción encontramos diferentes presentaciones clínicas. Cuando es unilateral los pacientes tienden a tener laterognasia con clase II dentaria ipsilateral y mordida abierta contralateral, mientras que en los casos que se presenta reabsorción condilar bilateral se caracteriza por una clase II dentaria, mordida abierta anterior progresiva y retrusión mandibular (Hoppenreijs et al., 1998, 1999; Gomes et al.).

En la evaluación de la reabsorción condilar es fundamental la imagenología. La telerradiografía de perfil y la radiografía panorámica son las imágenes de elección en los estudios observacionales (90,4 \%) (Merkx \& Van Damme; Kerstens et al.; de Mol van Otterloo et al.; Bouwman et al., 1994, 1997; De Clercq et al.; Scheerlinck et al.; Cutbirth et al.; Hoppenreijs et al., 1998, 1999; Hwang et al., 2000a,b, 2004; Wolford et al., 2002, 2003; Borstlap et al.; Wohlwender et al.; Kobayashi et al.; Nogami et al.). Sin embargo, al proveer imágenes en dos dimensiones y generar magnificación de ésta, es limitado su uso y el valor diagnóstico que poseen (Merkx \& Van Damme; Hwang et al., 2004). Por otro lado, el Cone Beam Computed Tomography (CBCT), permite una alta capacidad de resolución sin magnificación, logrando identificar la fase temprana de la reabsorción condilar (Xi et al.; Gomes et al.). El CBCT permite evaluar cambios dimensionales en sentido lineal, angular y volumétrico de las estructuras craneofaciales con mayor exactitud (Xi et al.; Gomes et al.). En los artículos incluidos se consideró como reabsorción condilar los casos en que había pérdida de estructura condilar superior a los $283 \mathrm{~mm}^{3}$ del volumen condilar (Xi et al.). Por el contrario, Gomes et al. estableció como reabsorción condilar la pérdida de estructura ósea mayor a 1,5 mm en el contorno condilar. También se utilizaron otros exámenes con menor frecuencia como la tomografía lateral de ATM en los estudios de Wolford et al. $(2002,2003)$ y resonancia magnética nuclear (RMN) en el estudio de Nogami et al. en búsqueda de presencia de hiperplasia sinovial. Por último, se propone el uso de la cintigrafía (T-99m) para evaluar un proceso de reabsorción o remodelación condilar. Sin embargo, este no es utilizado en ninguno de los estudios analizados por esta revisión (Merkx \& Van Damme).

En conclusión, la literatura describe que pacientes de sexo femenino, cursando la década de los veinte años, clases II esqueletales con retrognasia o hipoplasia mandibular y alto ángulo del plano mandibular son pacientes susceptibles para desarrollar reabsorción condilar post cirugía ortognática. Se describe mayor riesgo en grandes avances mandibulares y cirugía bimaxilar u osteotomía sagital de rama mandibular. Los signos imagenológicos serían evidenciables a los 6 meses post cirugía, por lo que el seguimiento clínico radiográfico es esencial en los pacientes intervenidos. En general, se describe que el diagnóstico es logrado con el uso del CBCT, evidenciando cambios a nivel condilar de forma más temprana. Debemos considerar que los múltiples estudios incluidos en esta revisión son relativamente antiguos y la evidencia no es muy amplia, por lo que se necesita continuar investigando en esta materia y así aclarar y establecer parámetros claros para su diagnóstico, prevención y tratamiento.

Tabla II. Resúmen de criterios diagnósticos de reabsorción condilar mandibular.

\begin{tabular}{llll}
\multicolumn{1}{c}{ Faciales } & \multicolumn{1}{c}{$\begin{array}{c}\text { Clínicos } \\
\text { Oclusales }\end{array}$} & \multicolumn{1}{c}{ Articulares } & \multicolumn{1}{c}{ Imagenológicos } \\
\hline $\begin{array}{l}\text { Disminución altura facial } \\
\text { posterior }\end{array}$ & $\begin{array}{l}\text { Discrepancias oclusales post- } \\
\text { quirúrgicas } \\
\text { Duscrepancias oclusales } \\
\text { progresivas }\end{array}$ & $\begin{array}{l}\text { Ruidos articulares (click y y } \\
\text { crépito) }\end{array}$ & $\begin{array}{l}\text { Cambios volumétricos en el cóndilo } \\
\text { mandibular }\end{array}$ \\
$\begin{array}{l}\text { Retrusión mandibular } \\
\text { progresiva }\end{array}$ & Aumento en overjet & Lateralidades y protrusivas & $\begin{array}{l}\text { Alteración forma del cóndilo } \\
\text { mandibular } \\
\text { Disminución en altura del cóndilo } \\
\text { mandibular } \\
\text { Disminución en altura rama } \\
\text { mandibular }\end{array}$ \\
\hline
\end{tabular}


ZERENÉ, R. J.; ZILLERUELO, P. M. J.; VERGARA, I. M.; NOGUERA, P. A. \& SOLÉ, V. P. Condylar resorption as a postoperative complication in patients undergoing orthognathic surgery. Narrative review. Int. J. Odontostomat., 13(3):251-257, 2019.

ABSTRACT: Condylar resorption as a complication following orthognathic surgery is considered to cause dento-facial anomalies, relating to different pre and intra-operative factors. The aim of the research was to describe condylar resorption as a postoperative complication after orthognathic surgery. A review of the literature was made in four databases: PubMed, EBSCO, Trip database and Epistemonikos. The search was carried out without year limiting, articles in English and Spanish, including systematic reviews, observational studies and clinical trials. Exclusion criteria were applied for report cases, animal studies and articles that do not relate condylar resorption with orthognathic surgery. Quality of evidence and strength of the recommendations were assessed for the chosen studies. For this study 21 articles were selected following the inclusion criteria. The literature found reported that condylar resorption is a relatively frequent complication following orthognathic surgery (1.4-32 \% of frequency) and that it can be associated with several factors such as genre, age, dento-maxillary anomaly and surgical technique. Condylar resorption is a complication that we must consider in the planning of orthognathic surgery, in order to identify risk factors and patients who are more likely to present this post-surgical complication. Following surgery, strict follow-up is a key factor to determine early clinical and radiographic changes. Finally, further research is needed to establish stronger prevention and diagnostic criteria.

KEY WORDS: orthognathic surgery, mandibular condyle, temporomandibular joint.

\section{REFERENCIAS BILIOGRÁFICAS}

Alexiou, K.; Stamatakis, H. \& Tsiklakis, K. Evaluation of the severity of temporomandibular joint osteoarthritic changes related to age using cone beam computed tomography. Dentomaxillofac. Radiol., 38(3):141-7, 2009.

Borstlap, W. A.; Stoelinga, P. J.; Hoppenreijs, T. J. \& van't Hof, M. A Stabilisation of sagittal split advancement osteotomies with miniplates: a prospective, multicentre study with two-year follow-up. Part III--condylar remodelling and resorption. Int. J. Oral Maxillofac. Surg., 33(7):649-55, 2004.

Bouwman, J. P.; Kerstens, H. C. \& Tuinzing, D. B. Condylar resorption in orthognathic surgery. The role of intermaxillary fixation. Oral Surg. Oral Med. Oral Pathol., 78(2):138-41, 1994.

Bouwman, J. P.; Tuinzing, D. B.; Kostense, P. J.; van Teeseling, R. A. \& Mokhtari, $\mathrm{H}$. The value of long-term follow-up of mandibular advancement surgery in patients with a low to normal mandibular plane angle. Mund Kiefer Gesichtschir., 1(6):311-5, 1997.

Cutbirth, M.; Van Sickels, J. E. \& Thrash, W. J. Condylar resorption after bicortical screw fixation of mandibular advancement. J. Oral Maxillofac. Surg., 56(2):178-82, 1998.

De Clercq, C. A.; Neyt, L. F.; Mommaerts, M. Y.; Abeloos, J. V. \& De Mot, B. M. Condylar resorption in orthognathic surgery: a retrospective study. Int. J. Adult Orthodon. Orthognath. Surg., 9(3):233-40, 1994.

de Mol van Otterloo, J. J.; Dorenbos, J.; Tuinzing, D. B. \& van der Kwast, W. A. TMJ performance and behaviour in patients more than 6 years after Le Fort I osteotomy. Br. J. Oral Maxillofac. Surg., 31(2):83-6, 1993.

Fish, L. C.; Epker, B. N. \& Sullivan, C. R. Orthognathic surgery: the correction of dentofacial deformities. J. Oral Maxillofac. Surg., 51(1 Suppl. 1):2841, 1993.
Gomes, L. R.; Cevidanes, L. H.; Gomes, M. R.; Ruellas, A. C.; Ryan, D. P.; Paniagua, B.; Wolford, L. M. \& Gonçalves, J. R. Counterclockwise maxillomandibular advancement surgery and disc repositioning: can condylar remodeling in the long-term follow-up be predicted? Int. J. Oral Maxillofac. Surg., 46(12):1569-78, 2017.

Hoppenreijs, T. J.; Freihofer, H. P.; Stoelinga, P. J.; Tuinzing, D. B. \& van't Hof, M. A. Condylar remodelling and resorption after Le Fort I and bimaxillary osteotomies in patients with anterior open bite. A clinical and radiological study. Int. J. Oral Maxillofac. Surg., 27(2):81-91, 1998.

Hoppenreijs, T. J.; Stoelinga, P. J.; Grace, K. L. \& Robben, C. M. Long-term evaluation of patients with progressive condylar resorption following orthognathic surgery. Int. J. Oral Maxillofac. Surg., 28(6):411-8, 1999.

Hwang, S. J.; Haers, P. E. \& Sailer, H. F. The role of a posteriorly inclined condylar neck in condylar resorption after orthognathic surgery. J. Craniomaxillofac. Surg., 28(2):85-90, 2000a.

Hwang, S. J.; Haers, P. E.; Seifert, B. \& Sailer, H. F. Non-surgical risk factors for condylar resorption after orthognathic surgery. J. Craniomaxillofac. Surg., 32(2):103-11, 2004.

Hwang, S. J.; Haers, P. E.; Zimmermann, A.; Oechslin, C.; Seifert, B. \& Sailer, H. F. Surgical risk factors for condylar resorption after orthognathic surgery. Oral Surg. Oral Med. Oral Pathol. Oral Radiol. Endod., 89(5):542$52,2000 b$.

Jung, H. D.; Jung, Y. S.; Park, J. H. \& Park, H. S. Recovery pattern of mandibular movement by active physical therapy after bilateral transoral vertical ramus osteotomy. J. Oral Maxillofac. Surg., 70(7):e431-7, 2012.

Kerstens, H. C. J.; Tuinzing, D. B.; Golding, R. P. \& van der Kwast, W. A. M. Condylar atrophy and osteoarthrosis after bimaxillary surgery. Oral Surg. Oral Med. Oral Pathol., 69(3):274-80, 1990.

Kobayashi, T.; Izumi, N.; Kojima, T.; Sakagami, N.; Saito, I. \& Saito, C. Progressive condylar resorption after mandibular advancement. Br. J. Oral Maxillofac. Surg., 50(2):176-80, 2012.

Merkx, M. A. \& Van Damme, P. A. Condylar resorption after orthognathic surgery. Evaluation of treatment in 8 patients. J. Craniomaxillofac. Surg., 22(1):53-8, 1994

Nogami, S.; Yamauchi, K.; Satomi, N.; Yamaguchi, Y.; Yokota, S.; Abe, Y. \& Takahashi T. Risk factors related to aggressive condylar resorption after orthognathic surgery for females: retrospective study. Cranio, 35(4):2508, 2016.

Scheerlinck, J. P.; Stoelinga, P. J.; Blijdorp, P. A.; Brouns, J. J. \& Nijs, M. L. Sagittal split advancement osteotomies stabilized with miniplates. A 2-5year follow-up. Int. J. Oral Maxillofac. Surg., 23(3):127-31, 1994.

Te Veldhuis, E. C.; Te Veldhuis, A. H.; Bramer, W. M.; Wolvius, E. B. \& Koudstaal, M. J. The effect of orthognathic surgery on the temporomandibular joint and oral function: a systematic review. Int. J. Oral Maxillofac. Surg., 46(5):554-63, 2017.

Wohlwender, I.; Daake, G.; Weingart, D.; Brandstätter, A.; Kessler, P. \& Lethaus, B. Condylar resorption and functional outcome after unilateral sagittal split osteotomy. Oral Surg. Oral Med. Oral Pathol. Oral Radiol. Endod., 112(3):315-21, 2011.

Wolford, L. M.; Karras, S. \& Mehra, P. Concomitant temporomandibular joint and orthognathic surgery: a preliminary report. J. Oral Maxillofac. Surg. 60(4):356-62, 2002

Wolford, L. M.; Reiche-Fischel, O. \& Mehra, P. Changes in temporomandibular joint dysfunction after orthognathic surgery. J. Oral Maxillofac. Surg., 61(6):655-60, 2003

Xi, T.; Schreurs, R.; van Loon, B.; de Koning, M.; Bergé, S.; Hoppenreijs, T. \& Maal, T. 3D analysis of condylar remodelling and skeletal relapse following bilateral sagittal split advancement osteotomies. J. Craniomaxillofac. Surg., 43(4):462-8, 2015.

Dirección para correspondencia:

Pedro Solé Ventura

Universidad de los Andes

Monseñor Álvaro del Portillo 12455

Las Condes

Santiago

CHILE

Recibido : 27-06-2018

Aceptado: 25-10-2018

E-mail: drpedrosole@gmail.com 